ARTICLE

\title{
A New Approach for Building an Atomic Model from a Three-Dimensional Electron Microscopy Data
}

\author{
Atsushi MATSUMOTO* \\ Japan Atomic Energy Agency, 8-1-7, Umemidai, Kizugawa, Kyoto 619-0215 Japan
}

\begin{abstract}
We have developed a new approach to build an atomic model from a low-resolution three-dimensional electron microscopy data for analyzing the structures of 70S ribosome. In this approach, a huge number of atomic models with different conformations are prepared first, and then the best-fitting atomic model is selected from them for each electron microscopy structure. The rigid-body fitting calculation, in which the atomic model is moved as a rigid body by translational and rotational manipulation, is most intensively performed. However, the calculation takes quite long if all the six rigid-body parameters are treated as variables at the same time. Thus, we perform two different calculations alternatively, in which only three parameters are treated as variables and the others are fixed. We have ascertained that the calculation is fast and accurate enough.
\end{abstract}

KEYWORDS: ribosome, normal mode analysis, rigid-body fitting, electron microscopy, atomic model, elastic network model

\section{Introduction}

Ribosome is a huge macromolecular complex responsible for protein synthesis in all organisms. There are four essential steps during protein synthesis in ribosome; initiation, elongation, termination, and recycling. In the initiation step, large 50S subunit and small 30S subunit come together, bind to mRNA and form an initiation complex of the 70S ribosome. In the elongation step, ribosome synthesizes proteins by translating the base sequence information on mRNA to the amino-acid sequence. In the termination step, protein synthesis is terminated and the newly synthesized peptide chain is released. Finally, in the recycling step, ribosome dissociates into two subunits for a new cycle of protein synthesis.

To understand the molecular mechanism of the protein synthesis, it is important to know the three-dimensional (3D) structures of ribosome in functional states. So far, the structures of ribosome in a few functional states are determined at the atomic resolution by X-ray crystallography. However, they are not enough for understanding the whole molecular mechanism of protein synthesis.

Recent progress in electron microscopy (EM) has made it possible to capture $3 \mathrm{D}$ structures of ribosome at various functional states. From the comparison of these EM structures, functionally important conformational changes have been identified. ${ }^{1)}$ However, because of the low resolution nature of the 3D-EM structures, it is not so easy to compare them directly. The atomic model, which is described by the positions of constituting atoms, is more suited for structural comparison. Thus, we have built an atomic model for each EM structure of ribosome. The results about the comparison

*Corresponding author, E-mail:matsumoto.atsushi@jaea.go.jp of the 42 structures were reported in our previous paper. ${ }^{2)}$ In this paper, the methodology for building the atomic model is described in detail.

\section{Method}

\section{Building EM Structure from EM Density Data}

The data for 3D-EM structure is available from the Electron Microscopy DataBank (EMDB, http://emdatabank.org) at the European Bioinformatics Institute (EBI). It is given as the density $\rho$ at each voxel, which occupies a space $(x, y, z)$, where $i_{x} p \leq x<\left(i_{x}+1\right) p, \quad i_{y} p \leq y<\left(i_{y}+1\right) p$, $i_{z} p \leq z<\left(i_{z}+1\right) p$, and $i_{x}, i_{y}$ and $i_{z}$ are integers, and $p$ is the voxel size given as an experimental value. From the data, we build an EM structure as follows. We define that each EM structure is composed of voxels whose density $\rho$ is higher than a threshold value $\rho_{0}$. The threshold value $\rho_{0}$ is determined so that the volume occupied by the EM structure ( $=N_{v} p^{3}$, where $N_{v}$ is the number of voxels with a density higher than $\rho_{0}$ ) is equivalent to the molecular volume of the X-ray crystal structure of the $70 \mathrm{~S}$ ribosome, calculated by a program VOIDOO ${ }^{4-6)}$ in which the probe radius is set to $1.4 \AA$.

\section{New Approach for Building Atomic Model from EM Structure}

Our approach to build an atomic model from each EM structure consists of two steps. In the first step, we build many atomic models with different conformations as a library by deforming the X-ray crystal structure. In the second step, the best-fitting atomic model is selected from them for each EM structure by rigid-body fitting calculation.

In the usual approach, which we call the "tailor-made" approach, time-consuming calculation for deforming the 
X-ray crystal structure so that it fits better into the EM structure is performed for each EM structure. On the other hand, in our "ready-made" approach, once the library of the deformed atomic models is prepared, only the rigid-body fitting calculation is necessary which require much less computational time. When we study many EM structures of the same target, our approach can save computational time.

\section{Atomic Model Library}

To build deformed atomic models, we employ normal mode analysis. In the normal mode analysis, molecular deformation can be described by a sum of normal modes;

$$
\mathbf{r}-\mathbf{r}_{0}=\sum_{k} \alpha_{k} \mathbf{v}_{0}^{k}
$$

where $\mathbf{r}$ and $\mathbf{r}_{0}$ are $3 N$-dimensional vectors, where $N$ is the number of atoms in the molecule, describing the deformed and energy-minimized atomic structures, respectively, $\mathbf{v}_{0}{ }^{k}$ is the $k$ th normal mode vector for the structure $\mathbf{r}_{0}$, and $\alpha_{k}$ the coefficient. In this study, we use Hookean pairwise potential as an energy function $E$;

$$
E=1 / 2 \sum_{(i, j)} C\left(d_{i j}-d_{i j}^{0}\right)^{2},
$$

where $d_{i j}$ is the distance between atom $i$ and $j$, the zero superscript indicates the initial structure, and $C$ is the coefficient. Note that the energy function is defined in such a way that the initial structure has the minimum energy. The summation is taken over all pairs of atoms $(i, j)$, whose distances in the initial structure are less than a cutoff distance, which depends on the atom types. Due to the form of this energy function, the model is called an elastic network model.

Equation (1) is a good approximation only when the deformation $\alpha_{k}$ is small. When it is large, the molecule does not deform along the low-energy path. Using an elastic network model, Miyashita et al. ${ }^{7)}$ showed that atomic structures with large deformations along the low-energy path could be obtained by applying small deformations along the normal mode iteratively. In a similar way, we obtain a series of deformed atomic structures $\mathbf{r}_{n}^{k}$ along the $k$ th normal mode of the X-ray crystal structure as follows; We start from the $\mathrm{X}$-ray crystal structure $\mathbf{r}_{0}$ and perform the normal mode analysis, in which the structure $\mathbf{r}_{0}$ is regarded as the initial structure in Eq. (2). Using the normal mode vector $\mathbf{v}_{0}^{k}$ $\left(\left|\mathbf{v}_{0}^{k}\right|=1\right)$ for the $k$ th lowest frequency normal mode, a deformed atomic structure $\mathbf{r}_{1}^{k}$ is obtained as $\mathbf{r}_{0}+\alpha \mathbf{v}_{0}^{k}$, where $\alpha(>0)$ is a constant and determines how far the structure is deformed. If we further regard the deformed structure $\mathbf{r}_{1}^{k}$ as the initial structure in Eq. (2), another normal mode analysis can be performed and normal mode vectors are obtained. Among them, the one $\mathbf{v}_{1}^{k_{1}}$ which is most similar to $\mathbf{v}_{0}^{k}$ $\left(\mathbf{v}_{0}^{k} \cdot \mathbf{v}_{1}^{k_{1}} \square 1\right)$ is used to build the next structure $\mathbf{r}_{2}^{k}$ $\left(=\mathbf{r}_{1}^{k}+\alpha \mathbf{v}_{1}^{k_{1}}\right)$. Note that $k_{1}$ is not always equal to $k$, because the order of the normal modes could be different for the deformed structure. The calculation is repeated, and a series of structures $\quad\left\{\quad \mathbf{r}_{n}^{k} ; n=1, \ldots, n_{+} \quad\right\}, \quad$ where $\mathbf{r}_{n}^{k}=\mathbf{r}_{0}+\alpha \mathbf{v}_{0}^{k}+\alpha \mathbf{v}_{1}^{k_{1}}+\alpha \mathbf{v}_{2}^{k_{2}}+\ldots+\alpha \mathbf{v}_{(n-1)}^{k_{(n-1)}}$, is obtained. Another series of structures is built for the opposite direction, that is, starting from the X-ray crystal structure $\mathbf{r}_{0}$, a deformed structure $\mathbf{r}_{-1}^{k}\left(=\mathbf{r}_{0}-\alpha \mathbf{v}_{0}^{k}\right)$ is built. The calculation is repeated and another series of structures $\left\{\mathbf{r}_{-n}^{k} ; n=1, \ldots, n_{-}\right\}$is obtained. We refer to these two series of structures $\left(\mathbf{r}_{n}^{k}\right.$ and $\mathbf{r}_{-n}^{k}$ ) as deformed structures along the $k$ th normal mode. In this study, we set the value of the constant $\alpha$ to 0.1 , which means that the root-mean-square displacement (RMSD) between $\mathbf{r}_{n-1}^{k}$ and $\mathbf{r}_{n}^{k}$ is $0.1 \AA$.

Using the deformed atomic structures $\mathbf{r}_{n}^{k}$, we replace Eq. (1) with

$$
\mathbf{r}-\mathbf{r}_{0}=\sum_{k}\left(\mathbf{r}_{n_{k}}^{k}-\mathbf{r}_{0}\right)
$$

for large molecular deformation. Note that $\mathbf{r}$ in Eq. (1) is determined by real numbers $\left(\alpha_{1}, \alpha_{2}, \alpha_{3}, \ldots\right)$, while $\mathbf{r}$ in Eq. (3) is by integers $\left(n_{1}, n_{2}, n_{3}, \ldots\right)$. Thus, a ready-made atomic structure $\mathbf{r}$ in Eq. (3) is specified by the combination of the integers $\left(n_{1}, n_{2}, n_{3}, \ldots\right)$.

\section{Scoring Function}

To build the best-fitting atomic model from each EM structure, a scoring function is necessary. The scoring function is an index to describe quantitatively how well each atomic model is fitted into the EM structure. We define the scoring function as a ratio of the number of atoms inside the space occupied by the EM structure to the total number of atoms. Usually, more refined scoring functions are used in the EM fitting calculations. ${ }^{8)}$ Our simple scoring function is chosen mainly for the fast rigid-body fitting calculation.

The scoring function is used for two purposes. Given an EM structure and an atomic model, the scoring function is maximized by the rigid-body fitting calculation to find the optimum position and orientation of the atomic model with respect to the EM structure. Then, the atomic model with the highest fitting score among the atomic models in the library is regarded as the best-fitting atomic model for the EM structure.

\section{Rigid-Body Fitting Calculation}

In the rigid-body fitting calculation, ${ }^{8)}$ the EM structure is fixed in space and only the atomic model is moved by rotational and translational manipulation. We perform two different calculations successively for the rigid-body fitting. In the first calculation, the atomic model is moved so that the principal axes of inertia for the atomic model and the EM structure overlap each other. If the two rigid bodies were exactly the same, this manipulation would make them overlap each other perfectly. Of course, they are not the same, and the position and orientation of the atomic model is further refined in the second calculation, where the atomic model is iteratively rotated and translated so that the fitting score is maximized. The details of the two calculations are described below. 
(1) Rigid-Body Fitting Using Principal Axes of Inertia

The inertial tensor $\mathbf{I}$ for an atomic model is described as follows;

$$
\mathbf{I}=\left(\begin{array}{ccc}
\sum m_{i}\left(y_{i}^{2}+z_{i}^{2}\right) & -\sum m_{i} x_{i} y_{i} & -\sum m_{i} x_{i} z_{i} \\
-\sum m_{i} y_{i} x_{i} & \sum m_{i}\left(x_{i}^{2}+z_{i}^{2}\right) & -\sum m_{i} y_{i} z_{i} \\
-\sum m_{i} z_{i} x_{i} & -\sum m_{i} z_{i} y_{i} & \sum m_{i}\left(x_{i}^{2}+y_{i}^{2}\right)
\end{array}\right)
$$

where $\left(x_{i}, y_{i}, z_{i}\right)$ is the position of atom $i$, and $m_{i}$ the mass. The origin of the coordinate system $\mathbf{o}$ is set on the center of mass of the atomic model. The principal axes of inertia $\left(\mathbf{e}_{x}, \mathbf{e}_{y}, \mathbf{e}_{z}\right)$ is obtained as the eigenvectors of the inertia tensor $\mathbf{I}$, that is, $\mathbf{I} \mathbf{e}_{x}=I_{1} \mathbf{e}_{x}, \mathbf{I} \mathbf{e}_{y}=I_{2} \mathbf{e}_{y}$, and $\mathbf{I} \mathbf{e}_{z}=I_{3} \mathbf{e}_{z}$, where $I_{1}, I_{2}$ and $I_{3}$ are inertial moments and we assume $I_{1} \leq I_{2} \leq I_{3}$. The inertial tensor $\mathbf{I}^{\prime}$ for an EM structure is similarly described, where $\left(x_{i}^{\prime}, y_{i}{ }^{\prime}, z_{i}^{\prime}\right)$ is the position of the center of each voxel, and we assume $m_{i}{ }^{\prime}$ is the same for all voxels. The origin $\mathbf{o}^{\prime}$ is set on the center of mass, and the principal axes of inertia $\left(\mathbf{e}_{x}{ }^{\prime}, \mathbf{e}_{y}{ }^{\prime}, \mathbf{e}_{z}{ }^{\prime}\right)$ satisfies $\mathbf{I}^{\prime} \mathbf{e}_{x}{ }^{\prime}=I_{l}{ }^{\prime} \mathbf{e}_{x}{ }^{\prime}, \mathbf{I}^{\prime} \mathbf{e}_{y}{ }^{\prime}=I_{2}{ }^{\prime} \mathbf{e}_{y}{ }^{\prime}$, and $\mathbf{I}^{\prime} \mathbf{e}_{z}{ }^{\prime}=I_{3}{ }^{\prime} \mathbf{e}_{z}{ }^{\prime}$, where $I_{1}{ }^{\prime} \leq I_{2}{ }^{\prime} \leq I_{3}{ }^{\prime}$.

The fitting calculation is performed by translational and rotational manipulation of the atomic model so that the origin $\mathbf{o}$ agrees with $\mathbf{o}^{\prime}$, and $\left(\mathbf{e}_{x}, \mathbf{e}_{y}, \mathbf{e}_{z}\right)$ with $\left(\mathbf{e}_{x}{ }^{\prime}, \mathbf{e}_{y}{ }^{\prime}, \mathbf{e}_{z}{ }^{\prime}\right)$. Because the directions of the principal axes cannot be defined uniquely (if $\mathbf{e}$ is an eigenvector of inertia tensor $\mathbf{I},-\mathbf{e}$ is also the eigenvector), there are four possible orientations for the atomic model, if we assume $\left(\mathbf{e}_{x}, \mathbf{e}_{y}, \mathbf{e}_{z}\right)$ and $\left(\mathbf{e}_{x}{ }^{\prime}, \mathbf{e}_{y}{ }^{\prime}, \mathbf{e}_{z}{ }^{\prime}\right)$ make right-handed systems. We calculate the fitting scores for the four orientations and choose the one with the highest score.

(2) Refinement of Rigid-Body Fitting by Iterative Rotation and Translation

In this calculation, the atomic position $\mathbf{r}_{i}\left(=\left(x_{i}, y_{i}, z_{i}\right)\right)$ is moved iteratively so that the fitting score is maximized. The atomic position $\mathbf{r}_{i}$ is described as

$$
\mathbf{r}_{i}=\mathbf{r}_{i}^{0}+\boldsymbol{\omega} \times \mathbf{r}_{i}^{0}+\boldsymbol{\delta},
$$

where $\mathbf{r}_{i}^{0}$ is the initial position, $\omega$ a rotation vector, around which each atom is rotated, and $\delta$ a translation vector. The vectors $\omega$ and $\delta$ are the variables and we perform the search for the optimum position and orientation of the atomic model by looking for the rotation and translation vectors which give the highest fitting score.

We start from the position $\mathbf{r}_{i}^{0}$ determined by the calculation using principal axes of inertia with the vectors $\omega$ and $\delta$ set to zero. At each repetition, the vectors $\omega$ and $\delta$ are changed to $\omega+\Delta \omega$ and $\delta+\Delta \delta$, respectively. We describe $\Delta \omega$ and $\Delta \delta$ as

$$
\begin{aligned}
& \Delta \boldsymbol{\omega}=a\left(l_{1}, l_{2}, l_{3}\right), \\
& \Delta \boldsymbol{\delta}=b\left(m_{1}, m_{2}, m_{3}\right),
\end{aligned}
$$

where $a$ and $b$ are the step sizes, and $\left(l_{1}, l_{2}, l_{3}, m_{1}, m_{2}, m_{3}\right)$ are integers. In this study, the range of $-3 \leq l_{q}, m_{q} \leq 3(q=1,2,3)$ is searched in each repetition. The combination of the integ- ers which gives the highest fitting score is selected and the vectors $\omega+\Delta \omega$ and $\delta+\Delta \delta$ are used as $\omega$ and $\delta$ in the next repetition. If all the selected integers are zero $(\Delta \omega=\Delta \delta=0)$, the step sizes $a$ and $b$ are made smaller. The iteration is terminated when $a$ and $b$ become small enough.

\section{Results and Discussion}

\section{Atomic Model Library}

We use three series of deformed structures $\mathbf{r}_{n}^{k}(k=5,6,7)$ for building atomic models by Eq. (3). The reason for choosing the three normal modes $(k=5,6,7)$ is described in our previous paper. $^{2)}$ We use 101 conformations $(-50 \leq n \leq 50)$ for $k=5$ and 7 , and 151 conformations $(-50 \leq n \leq 100)$ for $k=6$. Thus, the atomic model library consists of 1.54 million $(=101 \times 101 \times 151)$ atomic models with different conformations. For each EM structure, the best-fitting atomic model is selected from this library by the rigid-body fitting calculation.

\section{Rigid-Body Fitting Calculation}

In Table 1, the fitting scores obtained by various rigid-body fitting methods are shown for the 42 EM structures. In the second column, the scores $S_{0}$ for the X-ray crystal structure fitted into the EM structures by using the principal axes of inertia are shown. They are quite dependent on the EM structures and tend to be low for the EM structures with low resolution (data not shown).

Equation (5) shows that the atomic positions are described by the six rigid-body parameters. Thus, in the refinement process of the rigid-body fitting, the search for the optimum position and orientation of the atomic model with respect to the EM structure is made in the six-dimensional space. However, if we allow all the six parameters to vary in each repetition, the computation takes quite long as shown in the last column of Table 1, where the CPU time $T_{6}$ required for fitting the X-ray crystal structure into each EM structure is listed. This is because the sampling space is large, that is, the number of combinations of the integers in Eq. (6) is large.

For fast computation, we perform two types of repetitions alternately. In one repetition, we allow only translational parameters to vary and fix the rotational parameters, and vice versa in another repetition. In this way, we make the computation faster by performing the search in the three-dimensional space at each repetition. The CPU time required for the computation is listed as $T_{3}$ in the 6th column of Table 1, which is much shorter than $T_{6}$.

The fitting scores for the X-ray crystal structure by the two different iterative calculations are shown in Table 1 . The fitting score $S_{3}$ is obtained by the iterative calculation performed in the three-dimensional space at all repetitions, while $S_{6}$ in the six-dimensional space. The latter is shown as $S_{6}-S_{3}$ in the fourth column. As expected, $S_{6}$ tends to be higher than $S_{3}$ and the average difference is $(1.09 \pm 0.76) \times 10^{-4}$. It may seem strange that $S_{6}$ is smaller than $S_{3}$ for two cases (EM-1065 and 1363), because larger area is searched by the calculation in the six-dimensional space than that in the three-dimensional space. However, as long as the searching 
Table 1 Results of rigid-body fitting calculations for 42 EM structures.

\begin{tabular}{|c|c|c|c|c|c|c|}
\hline EM-ID & $S_{0}$ & $S_{3}$ & $\begin{array}{c}S_{6}-S_{3} \\
\left(\times 10^{-4}\right)\end{array}$ & $\begin{array}{c}\sigma \\
(\AA)\end{array}$ & $\begin{array}{c}T_{3} \\
(\mathrm{sec})\end{array}$ & $\begin{array}{c}T_{6} \\
(\mathrm{sec})\end{array}$ \\
\hline 1003 & 0.803 & 0.867 & 1.42 & 0.533 & 45 & 4377 \\
\hline 1004 & 0.509 & 0.735 & 0.08 & 0.108 & 58 & 4877 \\
\hline 1005 & 0.521 & 0.697 & 1.38 & 0.347 & 55 & 5878 \\
\hline 1006 & 0.743 & 0.833 & 0.69 & 0.180 & 52 & 4691 \\
\hline 1007 & 0.761 & 0.842 & 1.82 & 0.234 & 49 & 5016 \\
\hline 1008 & 0.770 & 0.839 & 0.53 & 0.222 & 40 & 5060 \\
\hline 1045 & 0.790 & 0.852 & 1.09 & 0.371 & 44 & 4350 \\
\hline 1055 & 0.634 & 0.806 & 0.36 & 0.112 & 47 & 4752 \\
\hline 1056 & 0.651 & 0.813 & 0.00 & 0.028 & 50 & 4648 \\
\hline 1064 & 0.470 & 0.666 & 2.31 & 0.267 & 50 & 5202 \\
\hline 1065 & 0.508 & 0.605 & -0.28 & 0.085 & 52 & 5630 \\
\hline 1068 & 0.485 & 0.802 & 2.75 & 0.477 & 61 & 7133 \\
\hline 1070 & 0.766 & 0.829 & 1.38 & 0.374 & 40 & 4446 \\
\hline 1071 & 0.594 & 0.753 & 1.09 & 0.191 & 48 & 4449 \\
\hline 1072 & 0.762 & 0.800 & 0.73 & 0.423 & 44 & 3736 \\
\hline 1077 & 0.777 & 0.825 & 1.05 & 0.192 & 47 & 4457 \\
\hline 1110 & 0.510 & 0.725 & 2.19 & 0.653 & 56 & 5560 \\
\hline 1122 & 0.622 & 0.823 & 1.22 & 0.328 & 46 & 4269 \\
\hline 1128 & 0.649 & 0.762 & 0.08 & 0.208 & 46 & 4657 \\
\hline 1143 & 0.647 & 0.853 & 1.05 & 0.344 & 47 & 4752 \\
\hline 1184 & 0.793 & 0.838 & 0.65 & 0.279 & 44 & 4406 \\
\hline 1185 & 0.744 & 0.815 & 1.94 & 0.599 & 47 & 4680 \\
\hline 1248 & 0.734 & 0.819 & 0.32 & 0.136 & 45 & 4406 \\
\hline 1250 & 0.640 & 0.757 & 0.36 & 0.057 & 41 & 4209 \\
\hline 1251 & 0.705 & 0.750 & 1.54 & 0.619 & 38 & 3963 \\
\hline 1261 & 0.458 & 0.801 & 0.81 & 0.064 & 71 & 6129 \\
\hline 1262 & 0.690 & 0.850 & 1.09 & 0.053 & 49 & 4735 \\
\hline 1263 & 0.765 & 0.875 & 0.57 & 0.172 & 47 & 4526 \\
\hline 1302 & 0.717 & 0.795 & 2.63 & 0.379 & 51 & 4609 \\
\hline 1310 & 0.821 & 0.843 & 1.98 & 0.437 & 45 & 4129 \\
\hline 1311 & 0.753 & 0.800 & 2.31 & 0.487 & 40 & 4137 \\
\hline 1312 & 0.736 & 0.827 & 1.46 & 0.139 & 43 & 4763 \\
\hline 1315 & 0.657 & 0.822 & 0.45 & 0.108 & 55 & 4940 \\
\hline 1323 & 0.558 & 0.716 & 0.81 & 0.297 & 56 & 4752 \\
\hline 1324 & 0.505 & 0.767 & 1.58 & 0.411 & 56 & 5344 \\
\hline 1362 & 0.675 & 0.810 & 1.09 & 0.081 & 54 & 4382 \\
\hline 1363 & 0.635 & 0.770 & -0.24 & 0.327 & 46 & 3981 \\
\hline 1365 & 0.623 & 0.784 & 1.50 & 0.494 & 47 & 5334 \\
\hline 1366 & 0.747 & 0.814 & 0.53 & 0.070 & 38 & 4255 \\
\hline 1370 & 0.688 & 0.803 & 1.86 & 0.227 & 48 & 4114 \\
\hline 1391 & 0.571 & 0.736 & 0.69 & 0.549 & 48 & 5320 \\
\hline 1395 & 0.781 & 0.829 & 0.85 & 0.079 & 45 & 4276 \\
\hline
\end{tabular}

area is limited (otherwise, the calculation takes forever), we can not avoid this happen.

In the fifth column, the average difference of the atomic position $\sigma\left(=\sqrt{\sum\left(\mathbf{r}_{i}^{(3)}-\mathbf{r}_{i}^{(6)}\right)^{2} / N}\right)$ is shown, where $\mathbf{r}_{i}^{(3)}$ and $\mathbf{r}_{i}^{(6)}$ are the $i$ th atomic position of the fitted atomic model by the calculations in the three and six-dimensional space, respectively. It is generally small and shows that the two fitted models are in the close position and orientation.

To see whether the difference $S_{6}-S_{3}$ is negligible or not, slightly deformed atomic models $\mathbf{r}_{1}^{k}(k=3,5,6)$, each of which is different from the X-ray crystal structure $\mathbf{r}_{0}$ by $0.1 \AA$ in RMSD, are fitted into each EM structure by iterative calculation in the six-dimensional space and the fitting scores $S_{6}\left(\mathbf{r}_{1}^{k}\right)$ are obtained. The average values of the magnitude of the difference $\left|S_{6}\left(\mathbf{r}_{1}^{k}\right)-S_{6}\left(\mathbf{r}_{0}\right)\right|$ for the $42 \mathrm{EM}$ structures are $(1.71 \pm 1.00) \times 10^{-4},(4.07 \pm 1.55) \times 10^{-4}$, and $(3.97 \pm 1.80) \times 10^{-4}$ for $k=3,5$, and 6 , respectively. These values are generally larger than the difference between the scores obtained by the calculations in the six and three-dimensional spaces (fourth column in Table 1), suggesting that the iterative calculation performed in the three-dimensional space at each repetition is practically accurate enough for selecting the best-fitting atomic model from the atomic model library.

\section{Conclusion}

We have developed a new approach to build the best-fitting atomic model from an EM structure and applied it for analyzing the EM structures of the $70 \mathrm{~S}$ ribosome. In our new approach, the rigid-body fitting calculation is most intensively executed, which is performed using the six rigid-body parameters. However, it takes quite long if all six parameters are treated as variables at the same time. Thus, we alternatively perform two different calculations, in which only three parameters are treated as variables and the others are fixed. We have ascertained that the calculation is fast and accurate enough.

\section{Acknowledgment}

This work was supported by Strategic International Cooperation Program and Core Research for Evolutional Science and Technology (CREST), Japan Science and Technology Agency and by Grants-in-Aid for Scientific Research on Priority Areas (No. 17053029) from the Ministry of Education, Culture, Sports, Science and Technology of Japan.

\section{References}

1) J. Frank, R. K. Agrawal, "A ratchet-like inter-subunit reorganization of the ribosome during translocation," Nature, 406[6793], 318-322 (2000).

2) A. Matsumoto, H. Ishida, "Global conformational changes of ribosome observed by normal mode fitting for 3D Cryo-EM structures," Structure, 17[12], 1605-1613 (2009).

3) L. Jenner, P. Romby, B. Rees, C. Schulze-Briese, M. Springer, C. Ehresmann, B. Ehresmann, D. Moras, G. Yusupova, M. Yusupov, "Translational operator of mRNA on the ribosome: how repressor proteins exclude ribosome binding," Science, 308[5718], 120-123 (2005).

4) G. J. Kleywegt, T. A. Jones, "Biomacromolecular Speleology," CCP4/ESF-EACBM Newsletter on Protein Crystallography 29, 
26-28 (1993).

5) G. J. Kleywegt, T. A. Jones, "Detection, delineation, measurement and display of cavities in macromolecular structures," Acta Crystallogr D Biol Crystallogr, 50[Pt 2], 178-185 (1994).

6) G. J. Kleywegt, J. Y. Zou, M. Kjeldgaard, T. A. Jones, "Around O," in Crystallography of Biological Macromolecules, edited by M.G. Rossmann, E. Arnold (Dordrecht: Kluwer Academic Publishers, The Netherlands, 2001), pp. 353-356,
366-367.

7) O. Miyashita, J. N. Onuchic, P. G. Wolynes, "Nonlinear elasticity, proteinquakes, and the energy landscapes of functional transitions in proteins," Proc. Natl. Acad. Sci. US A, 100[22], 12570-12575 (2003).

8) M. G. Rossmann, R. Bernal, S. V. Pletnev, "Combining electron microscopic with x-ray crystallographic structures," $J$. Struct. Biol., 136[3], 190-200 (2001). 